\title{
Editorial overview: lysosomal storage disorders with primary neurological involvement
}

\author{
Gregory M. Pastores • Derralynn A. Hughes
}

Published online: 15 June 2010

(C) SSIEM and Springer 2010

The focus of this issue of the Journal of Inherited Metabolic Disease is on lysosomal storage diseases (LSD) subtypes associated with primary neurological involvement. Conceptual advances that have had practical applications will be highlighted in this review issue.

Although rare, LSDs are a global problem and represent life-limiting or threatening disorders that can have a significant impact on patients' wellbeing. We have chosen experts from around the world, whose experience reflect the forefront in medical genetics devoted to progress in the LSDs.

In the course of soliciting articles on the topic, it became apparent that use of certain terms required clarification. Although several publications have described LSD variants with primary neurological involvement as neuropathic or neuronopathic forms of the disease, strictly speaking this choice of terms is misplaced. In our copy of Pryse-Phillips' Companion to Clinical Neurology (1995), neuropathy is defined as a disorder of one or more peripheral nerves, whereas neuronopathy is used to refer to a disease of the peripheral nervous system in which primary neuropathological changes appear in the nerve cell body rather than the axon of the myelin sheath. As peripheral nerve pathology is seen in only a subset of LSDs, the term neurodegenerative was suggested as a more appropriate descriptor, which may

\section{G. M. Pastores $(\bowtie)$}

Departments of Neurology and Pediatrics,

New York University School of Medicine,

New York, USA

e-mail: Gregory.Pastores@nyumc.org

D. A. Hughes

Department of Academic Haematology, Royal Free Campus, University College Medical School,

London, UK be acceptable but perhaps not for all entities (e.g., mucolipidosis IV). In the end, and for the sake of brevity, it was decided the term neuropathic would be retained, but discussion of disorders would not cover topics relating to secondary neurologic complications (e.g., radiculopathy secondary to vertebral compression in osteoporotic patients with Gaucher disease).

Over the past few decades, we have witnessed remarkable progress in our understanding of LSD; a group of inborn errors of metabolism characterized by a disruption in the degradation, processing and/or transport of various molecules that represent by-products of cellular turnover. The article by Jardim and colleagues, presented in two formats - a review in print and a detailed description of individual clinical entities in an electronic version as supplementary material-describes the various modes of clinical presentation. It is evident that, historically, examination of the phenotype has not included systematic analysis of disease course in ways that enable quantitative assignment of disease severity or rate of progression. Furthermore, studies that have attempted to correlate clinical expression with the genotype have repeatedly pointed to the lack of perfect concordance, except in a few cases, and there remain limited investigations of the factors that influence age of onset or specific patterns of neuronal vulnerability. Recent interest in the neurological problems noted in patients with Gaucher disease type 1 (GD1, conventionally viewed as the non-neuronopathic form) has prompted re-evaluation of the phenotype and investigations of putative causal links, if any, and in particular, the relationship between GD1 and risk for Parkinsonism and/or peripheral neuropathy. Along these lines, the paper by Cherin and co-workers describe observations garnered from a national (French) cross-sectional observational survey. 
In a separate paper, Tylki-Szymanska and colleagues describe the clinical features noted in patients with neuropathic Gaucher disease enrolled in a registry program, which underscores the role played by disease-specific registries in delineation of disease phenotypes. As the registry or surveillance programs represent post-approval commitments requested by regulatory agencies, the programs have been funded solely by the drug manufacturer. Although the registry programs include information on untreated patients, their focus has been on disorders for which treatment is available, and most of the published datasets have primarily centered on somatic aspects of disease. Given the rarity of these disorders, there will be limited opportunities for systematic data collection. Thus, it is hoped that most care-givers would actively participate in the design and implementation of registry-related programs.

The article by Bellettato and Scarpa provides a cogent description of relevant disease mechanisms, and calls attention to parallel changes between neurodegenerative LSDs presenting in children with disorders, such as Alzheimer, Huntington and Parkinson diseases, that are encountered in the elderly. Although the initiating biochemical basis for most subtypes has been well characterized, there is incomplete understanding of the cascade of events that lead to the complex phenotypes encountered in clinical practice. Overlap in presentation and disease course suggests there may be convergent pathologic mechanisms or common downstream targets. These observations are highlighted in the article by Hemsley and Hopwood who review insights that have been drawn from studies of several animal models of disease. It is hoped that lessons learnt as it relates to disease-related changes, in particular those which occur prior to development of overt signs and symptoms, will prove instructive on the appropriate time for intervention and the potential reversibility of the disease process.

The article by Schiffmann reviews current and emerging therapies for LSDs, and the particular challenges relating to drug delivery across the blood-brain barrier (BBB) into the central nervous system. A separate article on the BBB in LSDs had been solicited, but was not submitted in time to form part of the current issue.

As irreversible changes within the central nervous system may have set in by the time of diagnosis, the best prospects for an optimal outcome may only be realized through early detection. As most carrier individuals are often not aware of their carrier status and associated reproductive risks, the best prospects for case identification may be achieved primarily through population screening programs, focusing on the newborn period. This topic is reviewed by Hwu and colleagues, who draw attention to dilemmas associated with early diagnosis against the background of an inability to either predict with full certainty the clinical outcome or provide specific therapy. In the paper by Elleder and colleagues, data on disease prevalence within a defined population-residents of the Czech Republic - are presented, enabling comparisons with other studies of disease incidence from distinct populations.

As evident from the articles in this issue, the LSDs continue to generate new insights into basic molecular processes that inform us of pathomechanisms and therapeutic strategies with potential for wider application to other categories of genetic disease. Further advances aimed at improving patients' lives can be expected, as the tradition of discovery is sustained by the expanding number of investigators in the field. 